\title{
Lobbying directo: un análisis de las prácticas del sector hotelero con las instituciones políticas
}

DOI: 10.22403/UQROOMX/TYP08/02

\author{
Ramon Palau \\ Universitat de Girona \\ Santiago Forgas \\ Escola Universitària de Turisme \\ Mediterrani-Universitat de Girona
}

\section{$|M|$}

Resumen

La práctica del lobbying como estrategia para influir en las decisiones de los poderes públicos se ha ido complicando con la aparición de los organismos supraestatales, como la Unión Europea, hasta el punto de que buena parte de las decisiones que se toman en las instituciones europeas afectan directamente a la legislación de los Estados-nación. Sin embargo, el efecto frontera continúa dominando la mentalidad de las personas y de las empresas, puesto que, en determinados sectores, todavía se percibe como demasiado lejana la influencia de esas instituciones. Es el caso del sector turístico, y especialmente del subsector hotelero, que, a partir de los datos que depara esta investigación, basa más sus prácticas de lobbying directo en las instituciones políticas municipales y autonómicas que en las europeas, destacando de manera especial la diferencia en las prácticas de lobbying entre las cadenas hoteleras de propiedad familiar y las de propiedad no familiar.

Palabras |Lobbying, cadenas hoteleras, instituciones políticas, gobierno, CLAVE Unión Europea, turismo

Correos electrónicos: ramon.palaus@udg.edu·santi.forgas@mediterranig.com 


\section{Introducción}

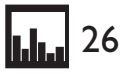

Teoría y Praxis

8 (20I0: 25-4I)
El lobbying como un instrumento utilizado para presionar e influir en la toma de decisiones de los responsables de las instituciones públicas - gobiernos, cámaras legislativas, administraciones públicas- ha sido ampliamente estudiado en Estados Unidos (Hunter, Wilson y Brunk, I 991; Hojnacki y Kimball, 1999; Victor, 2007; Shanagan et al, 2010) y en la Unión Europea (Bouwen, 2002 y 2004;Woll, 2006; Baumgartner, 2007; Coen, 2007). Sin embargo, la comunidad científica no se ha ocupado a suficiencia de estudiar el uso del lobbying en cuanto técnica de presión ante las instituciones públicas del sector turístico. Apenas algunos autores (Müller y Zaugg, 2005; McGehee, Meng y Tepanon, 2006) han realizado investigaciones que abordaban el fenómeno del lobbying como instrumento de política de empresa, o, muy recientemente, Gawande, Maloney y Montes, (2009) demuestran, a partir de modelos econométricos, la eficacia de las prácticas de lobbying para condicionar la opinión de los turistas hacia los destinos turísticos extranjeros. Asimismo, los cambios en las relaciones internacionales han planteado en algunas partes del mundo, como es el caso de la Unión Europea, la pérdida de valor de las fronteras de los Estadosnación hacia organizaciones políticas de carácter supraestatal con transferencias de competencias hacia la Unión que ha incrementado la actividad transfronteriza de los grupos de presión (Coen, 2007:336). Esto significa que, en el contexto europeo, las empresas han de desarrollar también, directa o indirectamente, sus actividades de presión ante las instituciones públicas, no sólo en sus Estadosnación, sino también desde una perspectiva transfronteriza, dirigiéndolas, más allá de sus fronteras nacionales, hacia quienes en Bruselas o Estrasburgo, sede de las instituciones europeas, van a tomar decisiones políticas que afectarán el funcionamiento de su actividad política, económica, social y cultural.

Este artículo se propone llevar a cabo una investigación exploratoria sobre la actividad de lobbying directo -término asimilable a técnica de presión de grupos de interés o de empresas y particulares- de las empresas hoteleras, separándolas especialmente según el tipo de propiedad, motivado por la importancia que en la economía europea tienen las empresas familiares. Así, 68 por ciento de las empresas catalanas (Callado et al., 2008: 19) y 60 por ciento de las europeas (IEF, 20I0: I) son negocios de propiedad familiar que, por su estructura de capital, por la presencia en los cargos ejecutivos de las familias propietarias, y por una estrategia empresarial a largo plazo -radicalmente distinto a las em- 
presas de propiedad no familiar, que basan su estrategia principal en el corto plazo-, tienen pautas de comportamiento diferenciadas de aquellas no familiares. Hemos dividido este artículo en diferentes apartados, donde los dos primeros son de conceptualización teórica y se refieren a lobbying y empresa familiar. En los siguientes apartados se expone la metodología empleada y los principales resultados obtenidos en la fase en que se recolectó la información. En los últimos se presentan las principales conclusiones y las implicaciones que los resultados sugieren para las empresas hoteleras, $y$ al final, las referencias bibliográficas utilizadas en el marco teórico.

\section{Lobbying}

Desde la aparición del fenómeno de la globalización de la economía y de los organismos supraestatales, las instituciones políticas se han convertido en un objetivo prioritario de las empresas. Hertz (200I: 74) considera que las ventajas competitivas ya no sólo se obtienen a partir de la clásica aportación de Porter (1987), es decir de la diferenciación de productos, los costes bajos y la especialización, sino ahora también convirtiéndose en grupo de presión con una estrategia política bien definida. Existe también una tradición en la investigación sobre los asuntos públicos en la que diversos autores (Vogel, 1979; Collins, 199|; Haug y Koppang, 1997; de Lange, 2000;Taminiau y Wilts, 2006) identifican, entre las estrategias que diseñan y ejecutan las empresas, las relaciones con las instituciones políticas y los partidos políticos. Ya en esa línea, Johnson (I991:7072) proponía con un cierto nivel de detalle, sin citar expresamente el término lobbying, esos dos públicos clave con los que las empresas debían relacionarse:

I. Administraciones públicas: las empresas han de identificar a las personas que puedan ser importantes en las diferentes administraciones, que, según Xifra (1998: 106), son los poderes Legislativo y Ejecutivo en los niveles local (municipio), intermedio (autonómico o regional en los países con descentralización autonómica o regional, o estado en los países federales), estatal (Estado-nación en los países autonómicos) o nacional (Estado-nación en los países federales con subdivisión por estados), y supraestatal -Unión Europea-, y que tengan la responsabilidad de tomar decisiones, para informarles de asuntos inmediatos que requieren la cooperación de los poderes públicos y, así, influir en sus decisiones. 
2. Partidos políticos: las empresas tienen que trabajar con todas las fuerzas políticas para sugerirles propuestas que tengan como consecuencia políticas cercanas a sus intereses.

Además, tal y como manifestaron Wilcox et al. (2000: 324), para determinar la importancia de las relaciones con las administraciones públicas y el poder político, las empresas necesitan de la administración actuaciones que ayuden a sus intereses, como permisos para construir edificios -caso habitual en hotelería-, propuestas para el ahorro de impuestos y la promulgación de leyes favorables. Es por esta razón que requieren desarrollar una estrategia de comunicación y de información especializada con el propósito de que aquellos que ejecutan las políticas públicas tomen las decisiones más favorables (Broscheid y Coen, 2007: 349). Para poder influir en los poderes públicos en un contexto como el europeo, cada vez más transfronterizo, donde buena parte de lo que se acabará convirtiendo en leyes en los Estados-nación ha sido previamente decidido en la Unión Europea a través de las decisiones, directivas y reglamentos emanados de las instituciones europeas (Comisión, Consejo y Parlamento), pero donde los Estados-nación y sus divisiones político-administrativas tienen todavía un peso muy significativo, se va imponiendo, poco a poco, la acción organizada del lobbying (Xifra, 1998:233). Para Attarça (1998:75), éste constituye una manera de promocionar el interés particular frente a los poderes públicos, con el objetivo final, según Xifra (1998:64), no siempre de procurar la modificación de una norma jurídica, sino frecuentemente también con el fin de relacionarse. La acción del lobbying se basa en poder influir en las decisiones de los poderes públicos de cualquier nivel de gobierno y, teniendo en cuenta que cada vez más las decisiones importantes se centran en el ámbito transfronterizo, sobre todo en las instituciones europeas, las presiones e influencias tienden a desplazarse más hacia el ámbito supraestatal y menos hacia el local, aunque las empresas siguen presionando mayormente a los poderes locales (Haug y Koppang, 1997: 236). De este modo, las empresas presionan a los poderes públicos (Hillman y Keim, 1995: 194), a través de sus propios medios; contratando a profesionales o agencias especializadas en lobbying, -los profesionales del lobbying, según McGrath (2005: I 25)-; o mediante los grupos de presión a los que pertenecen (Xifra, 1998: 85).

Si se revisa con mayor profundidad la literatura académica encontramos, entre los autores, que según Noguero (1995: 78 y 122) han contribuido al desarrollo de modelos que explican la teoría y las técnicas de relaciones públicas 
(Grunig y Hunt, I 984 y Cutlip, Center y Broom, 200 I) propuestas conceptuales diferentes. Grunig y Hunt (1984: 420), al igual que otros compiladores como Seitel (2002: 468-469) y Wilcox et al. (200I: 347-348), no tratan el lobbying como una estrategia individual sino que la incluyen en lo que denominan relaciones con el gobierno, asuntos públicos, información pública o comunicación pública. En cambio la traducción española de Cutlip, Center y Broom, (200 I:24I-244) sí tiene un apartado específico sobre la materia. Todos ellos, sin embargo, se ocupan ampliamente de los preceptos normativos que legislan la práctica del lobbying en Estados Unidos y exponen diferentes y muy variados instrumentos para llevar a cabo tal práctica, tanto técnicas de comunicación y de relaciones públicas, como prácticas escritas y técnicas orales. Entre las primeras destaca la elaboración de informes sobre temas específicos y los boletines informativos de empresas y asociaciones empresariales. Entre las orales, las visitas y reuniones con los públicos clave y las declaraciones públicas configuran, según estos autores, el núcleo más importante de las tácticas del lobbying. No obstante, para el caso de la Unión Europea, que presenta una gran complejidad de estructuras administrativas, Taminiau y Wilts (2006: I25) descubrieron que para que las prácticas de lobbying tengan éxito es necesario contar con un buen conocimiento y la información suficiente sobre las estructuras de la administración europea. Existe un número considerable de estrategias posibles de lobbying, pero quienes las han sintetizado mejor han sido Taminiau y Wilts (2006: I24), identificando tres estrategias de lobbying que las grandes empresas desarrollan:

I. Suministrar información y experiencia a los que toman las decisiones públicas: es una estrategia de lobbying directo que consiste en aportar información sobre la posición de las empresas en relación con las políticas públicas para influir en la toma de decisiones.

2. Apoyar económicamente a candidatos y partidos políticos: ésta es una práctica común en Estados Unidos pero no en Europa, donde la ausencia de legislación en muchos estados de la Unión ha originado corrupción política y financiación ilegal de partidos políticos, juzgados y condenados por los aparatos judiciales.

3. Grassroots lobbying: es una técnica indirecta y se trata de movilizar a un conjunto de personas que forman parte del grupo de interés, o que están vinculadas $o$ identificadas con quien tiene interés en que los poderes públicos modifiquen determinadas decisiones o regulaciones, para que los 
presionen; o bien se intenta la colaboración ciudadana para que se dirijan directamente a quien toma las decisiones sin que se sepa que tras las movilizaciones existe una organización o grupo que las manipula. Los canales habituales son los medios de comunicación para que expliquen el problema y lo incorporen en su agenda y las nuevas tecnologías de la información y la comunicación.

\section{Empresa familiar}

Para justificar la distinción que en este artículo se hace entre empresa familiar y no familiar hemos de señalar que las investigaciones en administración de empresas ya evidenciaron esa diferencia, sobre todo desde la última década del siglo pasado. Así, podemos destacar algunos autores que en la literatura académica constatan esa distinción, como Cooper, Upton y Seaman (2005) en management; Gudmundson, Hartman y Tower (1999) y Gallo,Tàpies y Cappuyns (2004) en orientación y estrategias de negocio; y Ensley y Pearson (2005) en las sinergias entre los equipos de alta dirección. Por esta razón, para diferenciar las empresas familiares de aquellas que no lo son, definiremos a estas últimas basándonos en las aportaciones de los autores que las han estudiado y siendo conscientes de la diversidad de definiciones existentes. En este sentido, para su investigación en Finlandia, Littunen y Hyrisky (2000: 4I) señalan de la manera más simple y contundente a la empresa familiar como aquella cuya propiedad está controlada por un propietario o por una familia propietaria, teniendo en cuenta que en sus conclusiones encontramos similitudes con las empresas familiares catalanas, puesto que concuerdan como una dedicación excesiva al mercado de proximidad, la orientación a largo plazo del negocio y su necesaria continuidad para proveer de ingresos a la familia. Sin embargo, como se ha expresado anteriormente, hay una gran diversidad de definiciones de empresa familiar, sin que poseamos todavía una única definición aceptada por la comunidad científica (Brockhaus, 1994; Litz, 1995; Pérez, 1998; Getz, Carlsen y Morrison, 2004) hasta el punto que Hugron (1998: 37) contabilizó 27, que iban desde la más restrictiva, que especifica que una empresa familiar ha de tener una propiedad exclusivamente familiar, hasta las que dicen que es suficiente considerar como familiar aquella empresa donde la familia influye en la dirección a través de métodos indirectos. 
Si empezamos por Leach (1991:22-23), encontraremos que definió la empresa familiar como aquella donde una familia o los vínculos familiares influyen y controlan sus decisiones, influencias queVilanova (1999:500) concretó en cuatro niveles: político o de poder, económico, ideológico y de continuidad. Gallo (1995: 13-14) y Gallo y García (1989: 68) utilizaron definiciones de diversos autores para sintetizar que una empresa es familiar cuando la mayoría del capital es detentado por una familia, cuando algunos miembros de esta familia trabajan en la empresa como directivos o como integrantes de los órganos de gobierno, y cuando la segunda generación de propietarios participa en los órganos de gestión. Sin embargo, tanto la definición de Gallo como la de Gallo y García nos parecen insuficientes puesto que no contemplan las propiedades de más de una familia, cuestión resuelta por Guinjoan y Llauradó (I998: I2-13) con una definición aplicable tanto a empresas que pertenecen a una sola familia, como las que están en poder de dos o más familias de una manera continuada en el tiempo, siempre y cuando el círculo familiar controle la gestión. Estos autores mencionan que por propiedad hemos de entender que la participación accionarial de la familia sea como mínimo mayoritaria -entre 50 y 80 por ciento del capital, como señalaron Bueno, Rodríguez y Camine, ( 1989 : I2)-, que la dirección tenga como mínimo a la familia en puestos de control y que exista una continuidad de la familia en la propiedad y en la dirección. Siguiendo esta misma línea argumental, Hugron (1998:37) y Bigné ( 1999: 18) coinciden en apuntar que una empresa es familiar cuando la propiedad y la gestión se transmiten bajo el control de los miembros de una familia con voluntad de continuidad familiar por medio de la incorporación de los descendientes en la propiedad y en la gestión. Por consiguiente, la empresa familiar, a pesar de sus múltiples definiciones, se diferencia claramente -y así lo consideramos en este artículo- de la no familar, porque son las familias propietarias quienes la gestionan, en contraposición con la empresa no familiar.

\section{Metodología}

El objetivo de esta investigación era identificar si las empresas desarrollaban estrategias de lobbying en las nuevas estructuras políticas transfronterizas. Para ello, y ante el poco conocimiento desarrollado en el sector turístico, realizamos una investigación exploratoria con un universo de empresas hoteleras 
que tuvieran un volumen significativo de negocio, que no fueran cadenas internacionales, $y$ cuyas oficinas centrales se ubican en Cataluña (España), con el fin de poder investigar dónde se tomaban las decisiones y encuestar a directivos con capacidad ejecutiva. El universo se concretó, a juicio de los investigadores, en cadenas hoteleras con cuatro o más hoteles, lo cual supuso la identificación de un universo de 38 cadenas hoteleras con un total de 675 hoteles, que representaban 25 por ciento del total de hoteles en Cataluña del censo de 2005. Se contactó telefónicamente con las 38 cadenas hoteleras identificadas, para entrevistar a un directivo (áreas de Mercadotecnia, Financiera y Dirección General) que contestara las preguntas de un cuestionario. Respondieron positivamente 24 cadenas hoteleras, que en su conjunto disponían de un total de 495 hoteles, que significaba 63.I por ciento de la totalidad de las cadenas que formaban el universo, 73.3 por ciento de todos los hoteles y 18.5 por ciento del total de hoteles en Cataluña.

El diseño del cuestionario fue con preguntas estructuradas y respuestas cerradas con escalas Likert con una valoración del I al 5 (I=poco o nada, $5=$ mucho o muy intenso), sometiéndolo a una prueba previa con expertos del sector turístico para que opinaran sobre la comprensión, categorización y disposición de las preguntas. Además, en el mismo cuestionario se adjuntaron otras con respuesta abierta que permitieran explorar mejor el objeto de estudio. La información, se recolectó entre los años 2005 y 2006 con base en la dispersión geográfica de la muestra. Una vez recopilada la información, se procedió a clasificar los datos y a analizar el material, obteniendo los resultados que se exponen en los apartados siguientes. Los datos de clasificación se presentan organizados en análisis univariable, el resto, en análisis bivariable, considerando las siguientes variables:

-Variables respuesta: organismos económicos y sociales, gobiernos, responsables de las prácticas de lobbying

- Variables explicativas: tipo de propiedad (familiar y no familiar), número de empleados (pequeñas: I-50 empleados; medianas: 5 I a 250; y grandes: 25 I o más). 


\section{Resultados}

Propiedad y tamaño

El cuadro I exhibe la propiedad de las 24 cadenas hoteleras de la muestra. Como puede observarse, 19 (79.2\%) son de propiedad familiar y cinco $(20.8 \%)$ de propiedad no familiar, lo cual nos da una proporción de empresas familiares más alta que la media catalana $(60 \%)$ y europea $(68 \%)$.

Si analizamos sólo las que son de propiedad familiar, en el cuadro 2 apreciaremos que, según el tamaño, todas se sitúan en las categorías de empresas medianas $(21.1 \%)$ y grandes $(78.9 \%)$.

La mayoría de las que son de propiedad no familiar también están en la categoría de empresas grandes (80\%), como podemos advertir enel cuadro 3.

Análisis de las prácticas de lobbying

CON ORGANISMOS ECONÓMICOS Y SOCIALES

En general, las empresas manifestaron que practican muy moderadamente actividades de lobbying y que son de tipo directo. En efecto, tal y como nos muestran las valoraciones del cuadro 4, podemos afirmar que las cadenas hoteleras desarrollan actividades moderadas. En particular, los organismos más significativos son las asociaciones empresariales no patronales (3.08) y los
Cuadro I. Cadenas HOTELERAS SEGÚN LA PROPIEDAD

\begin{tabular}{lcc}
\hline Propiedad & $\begin{array}{c}\text { Núm. } \\
\text { cadenas }\end{array}$ & (\%) \\
\hline Familiar & 19 & 79.2 \\
\hline No Familiar & 5 & 20.8 \\
\hline Total & 24 & 100.0 \\
\hline
\end{tabular}

Cuadro 2. Cadenas

HOTELERAS FAMILIARES POR EMPLEADOS

\begin{tabular}{l|c|c}
\hline Núm. empleados & $\begin{array}{c}\text { Núm. } \\
\text { cadenas }\end{array}$ & $(\%)$ \\
\hline $51-250$ & 4 & 21.1 \\
\hline 251 o más & 15 & 78.9 \\
\hline Total & 19 & 100.0 \\
\hline
\end{tabular}

Cuadro 3. Cadenas hoteleras NO FAMILIARES POR EMPLEADOS

\begin{tabular}{lcr}
\hline $\begin{array}{c}\text { Núm. } \\
\text { empleados }\end{array}$ & $\begin{array}{c}\text { Núm. } \\
\text { cadenas }\end{array}$ & $(\%)$ \\
\hline I-50 & I & 20.0 \\
\hline 25I o más & 4 & 80.0 \\
\hline Total & 5 & 100.0 \\
\hline
\end{tabular}


Lobbying directo: un análisis de las prácticas del sector hotelero con las instituciones políticas

parques feriales (3.04). El cuadro 4 expone la valoración que hacen las cadenas hoteleras en cuanto a la intensidad del lobbying con diversas entidades y organismos, utilizando el "tipo de propiedad" como variable explicativa. Observamos que, en general, las cadenas hoteleras familiares tienden a desarrollar más actividades de lobbying que las no familiares. Las medias más significativas son con las asociaciones empresariales ( 3.32 contra 2.20 ), entidades patronales ( 3.00 contra 2.00), cámaras de comercio ( 2.89 contra 2.20$)$, entidades económicas (2.37 contra 1.80$)$ y parques feriales ( 3.16 contra 2.60$)$. Cabe destacar que la relación con los partidos políticos es casi inexistente ( 1.58 de la valoración total).

Cuadro 4.VAloración de LA ACTIVIDAD DE LOBbying DE LAS CADENAS HOTELERAS CON ORGANISMOS ECONÓMICOS Y SOCIALES SEGÚN LA PROPIEDAD

Tipo de propiedad de las cadenas hoteleras

\begin{tabular}{|c|c|c|c|c|c|c|c|c|c|}
\hline Organismos & \multicolumn{3}{|c|}{$\begin{array}{l}\text { Familiar } \\
\text { Desv. }\end{array}$} & \multicolumn{3}{|c|}{$\begin{array}{l}\text { No Familiar } \\
\text { Desv. }\end{array}$} & \multicolumn{3}{|c|}{$\begin{array}{l}\text { Total } \\
\text { Desv. }\end{array}$} \\
\hline $\begin{array}{l}\text { Asociaciones } \\
\text { empresariales } \\
\text { no patronales }\end{array}$ & 3.32 & 1.003 & 4.00 & 2.20 & 1.304 & 2.00 & 3.08 & 1.139 & 4.00 \\
\hline $\begin{array}{l}\text { Entidades } \\
\text { patronales }\end{array}$ & 3.00 & 0.943 & 3.00 & 2.00 & 1.414 & 1.00 & 2.79 & 1.103 & 3.00 \\
\hline $\begin{array}{l}\text { Partidos } \\
\text { políticos }\end{array}$ & 1.58 & 0.769 & 1.00 & 1.60 & 0.894 & 1.00 & 1.58 & 0.776 & 1.00 \\
\hline $\begin{array}{l}\text { Cámaras de } \\
\text { comercio }\end{array}$ & 2.89 & 1.370 & 3.00 & 2.20 & 1.304 & 2.00 & 2.75 & 1.359 & 2.50 \\
\hline $\begin{array}{l}\text { Entidades } \\
\text { económicas }\end{array}$ & 2.37 & 1.165 & 2.00 & 1.80 & 0.837 & 2.00 & 2.25 & 1.113 & 2.00 \\
\hline $\begin{array}{l}\text { Parques } \\
\text { feriales }\end{array}$ & 3.16 & 1.015 & 3.00 & 2.60 & 1.140 & 3.00 & 3.04 & 1.042 & 3.00 \\
\hline $\begin{array}{l}\text { Grupos } \\
\text { ecologistas }\end{array}$ & 1.21 & 0.419 & 1.00 & 1.40 & 0.894 & 1.00 & 1.25 & 0.532 & 1.00 \\
\hline ONG'S & 1.89 & I.243 & 1.00 & 1.60 & 0.894 & 1.00 & 1.83 & 1.167 & 1.00 \\
\hline Otros & 1.53 & 0.905 & 1.00 & 1.40 & 0.894 & 1.00 & 1.50 & 0.885 & 1.00 \\
\hline
\end{tabular}




\section{Con gobiernos}

El cuadro 5 nos indica las prácticas de lobbying que desarrollan las empresas hoteleras con los diferentes niveles de gobierno. Podemos apreciar cómo son más fuertes en la proximidad (gobierno municipal) y más débiles cuanto más alejado está el gobierno de la institución.Así, observamos que la Unión Europea es con quien casi no se mantiene ninguna actividad. Si lo analizamos con un poco más de detalle, y tomamos el "tipo de propiedad" como variable explicativa, comprobaremos que las empresas familiares desarrollan una intensidad mayor que las no familiares, especialmente en los dos primeros niveles de la administración pública, el municipal (3.37 contra 2.40 ) que es algo más significativo que el autonómico (2.26 contra 2.00). Según puede cerciorarse, las empresas encuestadas han valorado como casi inexistentes el lobbying con las categorías Estado-nación e instituciones europeas.

Cuadro 5.VAloración de la actividad dE

LOBBYING DE LAS CADENAS HOTELERAS SEGÚN LA PROPIEDAD

Tipo de propiedad de las cadenas hoteleras

\begin{tabular}{|c|c|c|c|c|c|c|c|c|c|}
\hline Gobiernos & Media & $\begin{array}{c}\text { Familia } \\
\text { Desv. } \\
\text { típ. }\end{array}$ & Mediana & \multicolumn{3}{|c|}{ No Familiar } & \multicolumn{3}{|c|}{$\begin{array}{l}\text { Total } \\
\text { Desv. }\end{array}$} \\
\hline Municipal & 3.37 & 0.89 & 4.00 & 2.40 & 1.34 & 3.00 & 3.17 & 1.04 & 3.00 \\
\hline Autonómico & 2.26 & 1.14 & 2.00 & 2.00 & 1.00 & 2.00 & 2.21 & 1.10 & 2.00 \\
\hline Estado-Nación & 1.42 & 0.60 & 1.00 & 1.40 & 0.89 & 1.00 & 1.42 & 0.65 & 1.00 \\
\hline $\begin{array}{l}\text { Instituciones } \\
\text { Unión Europea }\end{array}$ & 1.26 & 0.56 & 1.00 & 1.40 & 0.89 & 1.00 & 1.29 & 0.62 & 1.00 \\
\hline
\end{tabular}

\section{RESPONSABLES DEL LOBBYING DIRECTO}

El cuadro 6 nos muestra la valoración de las cadenas hoteleras de propiedad familiar en cuanto a quién, dentro de su organigrama, desarrolla las actividades de lobbying directo. En general, puede advertirse que los directivos profesionales son los responsables de la ejecución del lobbying directo (3.53), por encima de los propietarios que participan en la gestión de la empresa (3.2I). Si hacemos 
el análisis utilizando el "número de empleados" como variable explicativa, encontramos que, en las grandes empresas (>250 empleados), directivos profesionales y propietarios ejecutivos obtienen la misma valoración (3.60); en cambio, en las medianas (5I-250 empleados), los directivos profesionales con una valoración muy superior (3.25) a la de los propietarios ejecutivos (1.75) son quienes ejecutan las actividades de lobbying directo.

Cuadro 6. Valoración de la Responsabilidad EN LA ACTIVIDAD

DE LOBBYING DE LAS CADENAS HOTELERAS SEGÚN EL NÚMERO DE EMPLEADOS

\begin{tabular}{|c|c|c|c|c|c|c|c|c|c|}
\hline \multicolumn{10}{|c|}{ Número de empleados } \\
\hline \multirow[b]{2}{*}{ Responsables } & \multicolumn{3}{|c|}{$5 \mathrm{I}-250$ empleados } & \multicolumn{3}{|c|}{$>250$ empleados } & \multicolumn{3}{|c|}{ Total } \\
\hline & Media & $\begin{array}{l}\text { Desv. } \\
\text { típ. }\end{array}$ & Mediana & Media & $\begin{array}{l}\text { Desv. } \\
\text { típ. }\end{array}$ & Mediana & Media & $\begin{array}{l}\text { Desv. } \\
\text { típ. }\end{array}$ & Mediana \\
\hline $\begin{array}{l}\text { Directivos } \\
\text { profesionales }\end{array}$ & 3.25 & 0.95 & 3.50 & 3.60 & 0.91 & 4.00 & 3.53 & 0.90 & 4.00 \\
\hline $\begin{array}{l}\text { Propietarios } \\
\text { ejecutivos }\end{array}$ & 1.75 & 1.50 & 1.00 & 3.60 & 1.05 & 4.00 & 3.21 & 1.35 & 4.00 \\
\hline $\begin{array}{l}\text { Familiares no } \\
\text { propietarios }\end{array}$ & 1.00 & 0.00 & 1.00 & 1.20 & 0.56 & 1.00 & 1.16 & 0.50 & 1.00 \\
\hline
\end{tabular}

\section{Conclusiones}

La investigación ha arrojado resultados relevantes en relación con la práctica del lobbying en las cadenas hoteleras catalanas objeto de estudio. Empero, al tratarse de una investigación exploratoria, no podemos generalizarlos. Primero, se observa el alto número de empresas familiares que existen en el subsector hotelero en Cataluña, muy superior, a efectos de esta muestra, a la mediana del país y a la mediana europea. Segundo, se confirma una moderada actividad en la práctica del lobbying de las empresas hoteleras hacia las organizaciones empresariales y los gobiernos municipales. Tercero, y sorprende, no se ha detectado lobbying hacia partidos políticos, considerando que son los representantes de estos partidos quienes, elegidos democráticamente por los electores, estarán en las instituciones públicas tomando decisiones sobre políticas públicas. Cuarto, se constata que las empresas de propiedad familiar desarrollan más actividades de lobbying que las de propiedad no familiar, propiciado, sin duda, porque los 
propietarios ejecutivos son quienes ejecutan el lobbying directo. Quinto, se ha evidenciado que, a pesar de la práctica desaparición de las fronteras comerciales y económicas en Europa, las empresas siguen mostrando mayor interés en ejercer presión sobre su gobierno más cercano, el municipal, que aunque tiene menos competencias que el resto de gobiernos se percibe como más próximo y accesible; lo cual coincide con las aportaciones de Haug y Koppang (1997:245) relativas a la importancia de ejercer prácticas de lobbying sobre las instituciones políticas geográficamente más próximas.A las cadenas hoteleras objeto de este estudio les quedan aún lejanas las instituciones transfronterizas para la práctica del lobbying directo o el indirecto, aunque, como ya se ha dicho, las directivas y reglamentos de la Unión Europea condicionan cada vez más las políticas locales.

\section{Implicaciones}

La evolución de las relaciones internacionales y del sistema financiero internacional en una economía internacionalizada, superando fronteras nacionales y eliminando barreras, habrá de repercutir forzosamente en las estrategias de las empresas. Una mayor actividad con las instituciones supraestatales y con los partidos políticos que las acaban configurando parece una línea de trabajo adecuada a seguir en los próximos años, puesto que, en el caso europeo, las decisiones competen cada vez más a las instituciones de la Unión Europea y menos a las de los Estados-nación. Como afirma Bouwen (2004:493), teniendo en cuenta que la práctica del lobbying ante las instituciones europeas está sometida a regulación, las empresas, si quieren tener éxito, deberán desarrollar sus actividades de lobbying considerando la legislación al uso. Por esta razón, las empresas, pero también los particulares, grupos cívicos, organizaciones ecologistas y sociales, deberán presionar más y con estrategias bien definidas y precisas ante la complejidad y el tamaño de las instituciones europeas, la globalización y lo intrincado de los problemas.

\section{FUENTES CONSULTADAS}

Attarça, M. (1998). "Affaires publiques: l'emergence d'une nouvelle fonction dans les enterprises". Revue Française de Gestion, I 19, 75-89.

Baumgartner, F.R. (2007).“EU Lobbying:AView from the US”.Journal of European Public Policy, I 4 (3), 482-488. 
Bigné, E. (1999). La empresa familiar. Retos de futuro. Valencia: Conselleria d’Economia, Hisenda i Administració Pública de la Generalitat Valenciana.

Bouwen, P. (2002). "Corporate Lobbying in the European Union:The Logic of Access". Journal of European Public Policy, 9 (3), 365-390.

(2004). "The Logic of Access to European Parliament: Business Lobbying in the Committee on Economic and Monetary Affairs". Journal of Common Market Studies, 42 (3), 473-495.

Brockhaus, R.H. (1994). "Entrepreneurship and Family Business Research: Comparisions, Critique, and Lessons". Entrepreneurship Theory and Practice, 19 (I), 25-38.

Broscheid, A. y D. Coen (2007). "Lobbying Activity and for a Creation in the EU: Empirically Exploring the Nature of the Police Good”.Journal of European Public Policy, I 4 (3), 346-365.

Bueno, E., J.M. Rodríguez y D. Camino (I989). "Características básicas de la empresa española: aspectos estructurales”. Papeles de Economía Española, 39, 2-18.

Callado, F., et al. (2008). Situació i perspectives econòmiques de l'empresa familiar a Catalunya (I). Gerona: Fundació Jaume Casademont.

Coen, D. (2007). "Empirical and Theoretical Studies in EU Lobbying”. Journal of European Public Policy, I 4 (3), 333-345.

Collins, M. (1991). A Complete Guide to European Research, Technology and Consultancy Funds. Guidelines for Succesful Applications, Lobbying,Acquisition and Use. Londres: Kogan Page.

Cooper, M.J., N. Upton y S. Seaman (2005).“Customer Relationship Management: A Comparative Analysis of Family and Nonfamily Business Practices". Journal of Small Business Management, 43 (3), 242-256.

Cutlip, S.M.,A.H. Center y G.M. Broom (200I). Relaciones públicas eficaces. Barcelona: Gestión 2000 [1952].

Ensley, M.D. y A.W. Pearson (2005). "An Exploratory Comparison of the Behavioral Dynamics of Top Management Teams in Family and Nonfamily New Ventures: Cohesion, Conflict, Potency, and Consensus”. Entrepreneurship Theory and Practice, mayo, 267-284.

Gallo, M.A. (1995). Empresa familiar. Textos y casos. Barcelona: Praxis.

Gallo, M.A. y C. García (1989). “La empresa familiar en la economía española”. Papeles de Economía Española, 39, 67-85. 
Gallo, M.A.,J.Tàpies y K. Cappuyns (2004).“Comparison of Family and Nonfamily Business: Financial Logic and Personal Preferences”. Family Business Review, I7 (4), 303-3I8.

Gawande, K.,W. Maloney y G. Montes (2009)."Foreign Informational Lobbying can Enhance Tourism: Evidence from the Caribbean”.Journal of Development Economics, 90, 267-275.

Getz, D., J. Carlsen y A. Morrison (2004). The Family Business in Tourism and Hospitality. Wallingford: Cabi.

Grunig, J. y T. Hunt (1984). Dirección de relaciones públicas. Barcelona: Gestión 2000.

Gudmundson, E., A. Hartman y C.B. Tower (1999). "Strategic Orientation: Differences between Family and Nonfamily Firms". Family Business Review, marzo, 27-39.

Guinjoan, M. y J.M. Llauradó (1998). “L'empresari familiar i el seu pla de successió”. Papers d'Economia, I0. Barcelona: Generalitat de Catalunya, Departament d'Indústria, Comerç i Turisme.

Haug, M. y H. Koppang ( 1997). "Lobbying and Public Relations in a European Context”. Public Relations Review, 23 (3), 233-247.

Hertz, N. (2002). El poder en la sombra. La globalización y la muerte de la democracia. Barcelona: Planeta.

Hillman, A.J. y G.D. Keim (1995). "International Variation in the Business-Government Interface-Institutional and Organizational Considerations". Academy of Management Review, 20 (I), 193-2I4.

Hojnacki, M. y D.C. Kimball (1999). "The Who and How of Organizations' Lobbying Strategies in Committee”. The Journal of Politics, 6I (4), 999-1024.

Hugron, P. (1998). “La régie d'entreprises familiales”. Gestion. Revue international de gestion, 23 (3), 37-40.

Hunter, K.G., L.A.Wilson y G.C. Brunk (199I). "Societal Complexity and Interest-Group Lobbying in the American States".Journal of Politics, 53 (2), 488-503.

IEF (20I0). La empresa familiar en el mundo. Instituto de la Empresa Familiar. Disponible en: www.iefamiliar.com/empresafam/datos.asp [2010, 29 de enero].

Johnson, H. ( 199 |). La gestión de la comunicación. Madrid: Ediciones de Ciencias Sociales. 
Lange, R. de (2000). "Public Affairs Practitioners in the Netherlands:A Profile Study”. Public Relations Review, 26 (I), 15-29.

Leach, P. (I99I). La empresa familiar. Barcelona: Omega.

Littunen, H. y K. Hyrisky (2000). "The Early Entrepreneurial Stage in Finnish Family and Nonfamily Firms". Family Business Review, I 3 (I), 4I-53.

Litz, R.A. (1995). "The Family Business: Towards Definitional Clarity”. Family Business Review, 8 (2), 9I-I07.

McGehee, N.G., F. Meng y Y. Tepanon (2006). "Understanding Legislators and their Perceptions of the Tourism Industry:The Case of North Carolina, USA, 1990 and 2003”. Tourism Management, 27, 684-694.

McGrath, C. (2005). "Towards a Lobbying Profession: Developping the Industry's Reputation, Education and Representation". Journal of Public Affaires, 5, I24-I 35.

Müller, H. y B. Zaugg (2005). “Lobbying in Swiss Tourism”. Tourism Review, 60 (4), 6-II.

Noguero, A. (1995). La función social de las relaciones públicas: historia, teoría y marco legal. Barcelona: EUB.

Pérez, LI. (1998). “La successió en l'empresa familiar catalana”, en M. Modest Guinjoan y J.M. Llauradó (eds.). La successió en l'empresa familiar catalana. Barcelona: Generalitat de Catalunya, Departament d'Indústria, Comerç i Turisme, I28-I 34.

Porter, M.E. (1987). Ventaja competitiva. México: Cecsa.

Seitel, F.P. (2002). Teoría y práctica de las relaciones públicas, $8^{a}$ ed. Madrid: Prentice Hall.

Shanagan, E.A., et al. (20I0). "From Protests to Litigation to YouTube: A Longitudinal Case Study of Strategic Lobby Tactic Choice for the Buffalo Field Campaign”. The Social Science Journal, 47, I37-I 50.

Taminiau, Y. y A. Wilts (2006). "Corporate Lobbying in Europe, Managing Knowledge and Information Strategies". Journal of Public Affaires, 6, I22- 130.

Victor,J.N. (2007)."Strategic Lobbying. Demonstrating How Legislative Context Affects Interest Groups' Lobbying Tactics”. American Political Research, 35 (6), 826-845.

Vilanova,A. (1999)."La empresa familiar: expectativas y retos en una sociedad de vértigo”. Alta Dirección, 208, 499-507. 
Vogel, D. (1979). Lobbying the Corporation: Citizen Challenges to Business Authority. Nueva York: Basic Books.

Wilcox, D.L., et al. (200I). Relaciones públicas. Estrategias y tácticas, $6^{\text {a }}$ ed. Madrid: Addison Wesley.

Woll, C. (2006)."Lobbying in the European Union:From Sui Generis to a Comparative Perspective”.Journal of European Public Policy, I 3 (3), 456-469.

Xifra, J. (1998). Lobbying. Cómo influir eficazmente en las decisiones de las instituciones públicas. Barcelona: Gestión 2000. 